\title{
Acanthus ebracteatus Vahl. Ethanol Extract Enhancement of the Efficacy of the Collagen Scaffold in Wound Closure: A Study in a Full-Thickness-Wound Mouse Model
}

\author{
Jutamas Somchaichana, ${ }^{1}$ Tanom Bunaprasert, ${ }^{2}$ and Suthiluk Patumraj ${ }^{1,3}$ \\ ${ }^{1}$ Inter-Department of Physiology, Graduate School and Faculty of Medicine, Chulalongkorn University, Bangkok 10330, Thailand \\ ${ }^{2}$ Laboratory of Tissue Engineering and Department of Otolaryngology, Faculty of Medicine, Chulalongkorn University, \\ Bangkok 10330, Thailand \\ ${ }^{3}$ Center of excellence for Microcirculation, Department of Physiology, Faculty of Medicine, Chulalongkorn University, \\ Bangkok 10330, Thailand
}

Correspondence should be addressed to Suthiluk Patumraj, suthilukp@yahoo.com

Received 4 July 2012; Revised 25 August 2012; Accepted 26 August 2012

Academic Editor: Rudi Beyaert

Copyright (C) 2012 Jutamas Somchaichana et al. This is an open access article distributed under the Creative Commons Attribution License, which permits unrestricted use, distribution, and reproduction in any medium, provided the original work is properly cited.

\begin{abstract}
Acanthus ebracteatus Vahl. is a Thai herb that is effective in wound healing. We sought to quantitatively determine whether or not the combined application of Acanthus ebracteatus Vahl. and a collagen scaffold will increase wound closure and angiogenesis. $\mathrm{Balb} / \mathrm{c}$ mice (body weight: $22-25 \mathrm{~g}$ ) were anesthetized with sodium thiopental. The dorsal skin incision measuring $1.5 \times 1.5 \mathrm{~cm}$ was made and then deepened using scissors to produce a full-thickness incision down to the level of the panniculus carnosus. The size of the wound was approximately $10 \%$ of the total body surface area. The collagen sheet was implanted onto the wound. Animals were divided into 4 major groups as follows: wound with normal saline (W-NSS), wound treated with $0.3 \mathrm{~g} / \mathrm{kg}$ BW of Acanthus ebracteatus Vahl. extract (W-AE $(0.3 \mathrm{~g} / \mathrm{kg} . \mathrm{bw})$ ), wound implanted with collagen scaffold (W-Coll), and wound implanted with collagen scaffold and treated with $0.3 \mathrm{~g} / \mathrm{kg}$ BW of Acanthus ebracteatus Vahl. (W-Coll-AE combination). On day 14, the W-Coll-AE group showed decreased wound areas and increased capillary vascularity (CV) when compared to the other 3 groups, W-NSS, $\mathrm{W}$-AE0.3, and W-Coll. In the present study, the combination of AE0.3 with collagen showed the best effect on skin angiogenesis and promoted wound closure with less neutrophil infiltration.
\end{abstract}

\section{Introduction}

Wound healing is a dynamic process that consists of four critical phases: hemostasis, inflammation, proliferation, and tissue remodeling [1]. In the proliferative phase, angiogenesis is an essential mechanism for healing wounds, restoring blood flow to tissues after injury, and achieving wound closure. In fact, the impairment of wound angiogenesis may lead to a prolonged period of wound healing $[2,3]$. Together with the inflammatory process, poor blood perfusion may hinder the dynamic process of wound healing and result in scar formation, especially in full-thickness wounds [4].

For the clinical treatment of full thickness wounds, several tissue-engineered skin substitutes have been developed. Among these tissue-engineered substitutes, the collagen scaffold, as shown by a number of clinical studies, has the greatest promise of being effective at promoting the regeneration of wound tissue. The collagen scaffold is a current technology that protects wounds from infection and dryness. In addition, the scaffold spacers are necessary for the enhancement and support of neovascularization [5].

Acanthus ebracteatus Vahl. is a member of the Acanthaceae family. Acanthus ebracteatus Vahl. has been used as folk medicine for a wide range of tropical diseases in Ayurveda. In India, whole plants are used as astringent, expectorant, and stimulant, the roots are used as a cough remedy, and the tender shoots and leaves are used as a snakebite cure [6]. In China, the stem and roots are useful against coughs, chronic fever, paralysis, asthma hepatomegaly, hepatitis, and lymphoma [7]. 
In Malaysia, its leaves and the boiled seeds are commonly used as a cough medicine [7]. Two or three seeds are used to treat intestinal worms in children $[7,8]$. The juice from leaves is used for preventing the hair loss. The roots are used against snake venom, and also to treat herpes zoster. The fruit pulp is used to poultice snake-bite wound [8].

In Philippines, the leaves and roots boiled are used in the form of a decoction against asthma [8]. The roots are applied with boiled milk, which is commonly used in leucorrhea and tonic drinks.

In Indonesia, the roots are used to poultice the wound that enhance the wound healing. In addition, the roots and ground ginger are taken together to salve the flatulence and treat the colic disease [8].

In Burma, the shoots are used against snake venom, and the leaves are used to treat the rheumatism [8].

In Vietnam, whole plants are used as diuretic agent, and the root is used to treat paralysis [8].

In Thailand, both genus of Acanthus spp., Acanthus ebracteatus Vahl., and Acanthus ilicifolius Linn. have been used in the same way as traditional medicine; the plant is used as a purgative and anti-inflammatory in arthritis [4]. The whole plant is boiled in water for bath in order to heal rash and skin diseases. The fresh plant is crushed and applied as a poultice in boils or taken orally as depurative. The fruits are taken orally to ease menstrual disorder [9].

Petroleum ether and alcoholic extracts of Acanthus ebracteatus Vahl. are reported for their therapeutic ingredients against inflammation, asthma, and skin diseases [10-12]. Beside, Laupattarakasem et al. [4] showed that aqueous extract of Acanthus ebracteatus Vahl. also exerts antiinflammatory effects by reducing thromboxane $\mathrm{B}_{2}\left(\mathrm{TXB}_{2}\right)$ and leukotriene $\mathrm{B}_{4}\left(\mathrm{LTB}_{4}\right)$. In 2005, Kongcharoensuntorn et al. [13] demonstrated antibacterial and antioxidant activities of desalinization of aqueous extract of Acanthus ebracteatus Vahl.

Interestingly, $\beta$-sitosterol, an active ingredient in Acanthus ebracteatus Vahl. extract was highlighted for its angiogenic properties similar to Aloe vera [14-16].

Moreover, in the extract from the stem of $A$. ebracteatus Vahl., bioactive polysaccharides, neutral, and acidic polysaccharides fractions have been reported for their effects of immunopotentiating, antitumor, antiangiogenic, and antioxidative activities [17-20].

Based on the beneficial effects of Acanthus ebracteatus Vahl. extract in enhancing angiogenesis, this study aims to investigate whether the topical application of Acanthus ebracteatus Vahl. extract can enhance the efficacy of the collagen scaffold in wound closure.

\section{Materials and Methods}

2.1. Animal Preparation. Male Balb/c mice aged 8-10 weeks and weighing $22-25 \mathrm{~g}$ were purchased from the National Laboratory Animal Center, Saraya Campus, Bangkok. The experimental procedures were conducted according to the National Research Council of Thailand guidelines for the use of experimental animals. The animals were housed in a pathogen limited room where the temperature was $25 \pm$ $3^{\circ} \mathrm{C}$. During the experiments, the animals were housed one animal per cage with free access to sterilized water and standard laboratory chow under a 12-hour light/dark cycle.

Four groups of mice were studied as follows: (1) wound with normal-saline-treated group (W-NSS, $n=5,0.9 \%$ normal saline (Sigma Chemical Co., USA) administered directly to the wound once daily until the end of experiment); (2) wound implanted with collagen scaffold (W-Coll, $n=5$ ); (3) wound treated with $0.3 \mathrm{~g} / \mathrm{kg}$ BW of Acanthus ebracteatus Vahl. extract (W-AE0.3, $n=5$ ); (4) wound implanted with collagen scaffold and treated with $0.3 \mathrm{~g} / \mathrm{kg}$. BW of Acanthus ebracteatus Vahl. extract (W-Coll-AE0.3, $n=5$ ). A sham group $(n=5)$, which consisted of normal mice without wounds, was used for comparison with W-NSS in the neutrophil infiltration experiment on day 3 of the study.

\subsection{Preparation of the Collagen Scaffold That Was Implanted} on the Wound. The collagen scaffold was prepared by $i$-Tissue Laboratory, Faculty of Medicine, Chulalongkorn University. Briefly, collagen type I (Sigma Chemical, USA) was mixed with $1 \%$ acetic acid, then molded and sterilized. A piece of $1.5 \times 1.5 \mathrm{~cm}$ collagen scaffold was used for each mouse after three 15-min sterilizations with PBS.

\subsection{Wound Creation and Collagen Scaffold Implantation.} In the groups in which wounds were created, the animals were anesthetized with thiopental (intraperitoneal injection, pentobarbital $20 \mu \mathrm{g} / \mathrm{mL}$ ) then their skin folds were shaved and disinfected with a noniodine-containing alcoholic skin disinfectant. Full-thickness skin defects measuring $2.25 \mathrm{~cm}^{2}$ $(1.5 \times 1.5 \mathrm{~cm})$ down to the muscle fascia were created; these were then extended down to the panniculus carnosus with scissors. One wound area was created for each mouse. The sterile collagen scaffold was placed onto the wound, precisely trimmed to the wound size, and fixed with multiple single stitches with 6-0 nylon suture [21].

2.4. Preparation of Acanthus ebracteatus Vahl. The ground stem $(5.0 \mathrm{~kg})$ of Acanthus ebracteatus was extracted with three treatments with $95 \%$ ethanol $(15.0 \mathrm{~L})$. The combined extract was filtered using filter paper (Whatman no. 1). The filtrate was evaporated under reduced pressure at $37^{\circ} \mathrm{C}$ to remove the ethanol. The concentrated extract was subsequently frozen and freeze-dried to yield $430 \mathrm{~g}$ of brown solid. These crude extracts were conducted by Dr. Sanya Hokputsa (Phytochemistry Research Laboratory, Government Pharmaceutical Organization, Bangkok, Thailand) [22]. In the W-AE0.3 group, this crude extract was mixed with normal saline and a final concentration of $0.3 \mathrm{~g} / \mathrm{kg}$ BW was applied topically to the wound area daily [23].

2.5. To Determine Wound Closure. On days 0, 7, and 14, the wound area of the animals was photographed using a digital camera at a working distance that was consistent for all animals; the wound area was analyzed by software (Image Pro II 6.1) as shown in Figure 1. Wound closure was determined by the percentage decrease in the wound area 


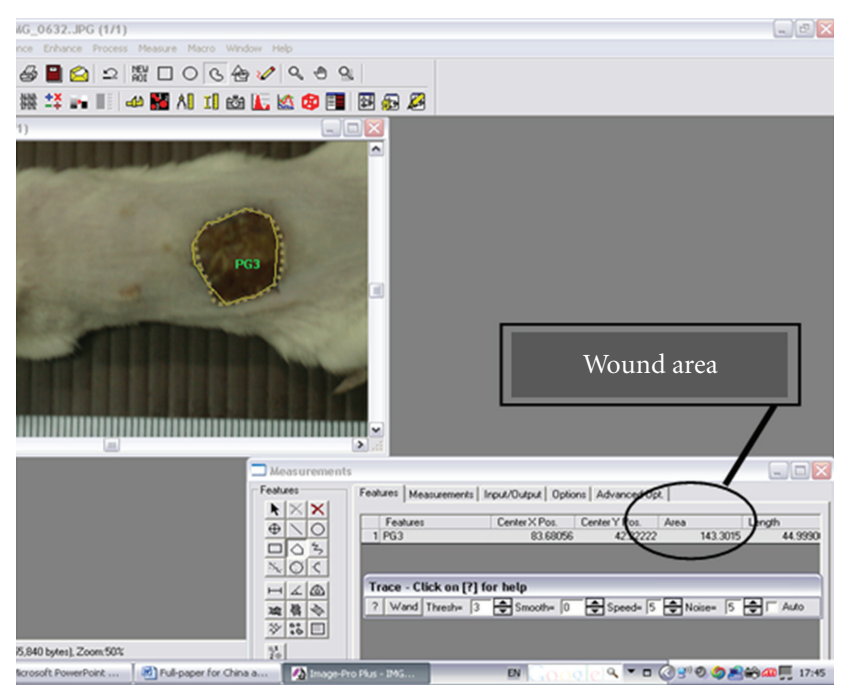

Figure 1: Wound area $\left(\mathrm{mm}^{2}\right)$ of each mouse was determined by using Image Pro II 6.1 software.

when compared to days 0 and 14 . Wound closure was then obtained by the following:

$\%$ Wound closure

$$
=\frac{\text { Wound } \operatorname{area} a_{\operatorname{day}(0)}-\text { Wound }_{\operatorname{area}} \mathrm{ady}_{(14)}}{\text { Wound } \operatorname{area}_{\mathrm{day}(0)}} \times 100 .
$$

2.6. To Determine Capillary Vascularity. Intravital fluorescence microscopy was used to assess the extent of capillary vascularity $(\mathrm{CV})$ in the wound area on days 7 and 14 in each group.

The equipment setup of the intravital fluorescence microscope consisted of a video microscope (Nikon E50i, Tokyo, Japan) with a 10x objective and eyepiece lens (Nikon, Japan), camera controller (Hamamatsu C2400, Japan), video timer (VTG-33, FOR-A, Japan), and video recorder (Panasonic NV-HD620, Japan). After anesthetization with thiopental (ip. $12 \mathrm{~g} / \mathrm{kg}$ BW pentobarbital), the intravenous injection of $0.2 \mathrm{~mL}$ of 5\% FITC-labeled dextran (MW.150000, Sigma Chemical Co., USA) was performed to guarantee contrast enhancement by staining of the plasma. The microscopic images were recorded on videotapes.

By using digital image processing software (Image Pro II 6.1), the microvessel diameters $(\mu \mathrm{m})$ and the CV were determined. From each video image, the region of interest (ROI) was selected with the approximate size of $100 \times 100$ pixels. Each ROI was selected to only cover a capillary network with a diameter of less than $10 \mu \mathrm{m}$. In each animal, 3 video images were obtained and approximately 3 ROIs were selected from each video image; therefore, approximately 9 values of CV were evaluated for each animal. By using Image Pro II Software, the total number of pixels that were located within a capillary network was determined (Figure 2) and divided by $100 \times 100$-pixel-ROI area. The $\% \mathrm{CV}$ that

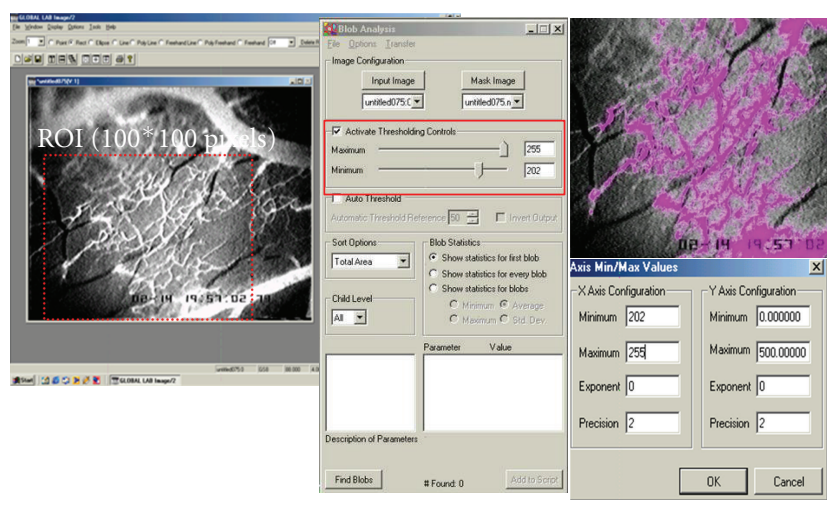

FIGURE 2: The total number of pixels located within the capillary network of each ROI $(100 \times 100$ pixels $)$ was determined by using Image Pro II software.

corresponded to capillary density [24-26] was defined as follows:

Capillary vascularity (\%)

$$
=\frac{\text { Number of pixels within capillaries }}{\text { Total number of pixels within the ROI }} \times 100 \text {. }
$$

2.7. To Determine Reepithelialization. After intravital fluorescence microscopy studies, the skin of the wounded area was collected, fixed in $4 \%$ phosphate-buffered formalin for 2-3 days, and then embedded in paraffin. From the paraffin-embedded tissue blocks, $5 \mu \mathrm{m}$ sections were serially cut and stained with hematoxylin-eosin ( $\mathrm{H}$ and $\mathrm{E})$ for routine histology. Reepithelialization was analyzed from the $\mathrm{H}$ and $\mathrm{E}$ slides of each group by using a stereo microscope, Nikon SMZ800. Reepithelialization was calculated as the ratio between the distance covered by the epithelium and the distance between wound ends.

$\%$ Reepithelialization

$$
=\frac{\text { Distance covered by epithelium }}{\text { Distance of the wound edges }} \times 100 \text {. }
$$

2.8. To Determine VEGF Levels in Wounded Tissue. To determine wounded tissue VEGF levels, a piece of skin was excised from the dorsal part of each mouse and frozen at $-80^{\circ} \mathrm{C}$. On the day of experiment, the tissue was rinsed with phosphate buffer solution (PBS) to remove excess blood, homogenized in $20 \mathrm{~mL}$ of PBS, and stored at $-20^{\circ} \mathrm{C}$. Two freeze-thaw cycles were performed to break the cell membranes; the homogenates were centrifuged for 5 minutes at $5000 \times \mathrm{g}$. Fifty $\mathrm{mL}$ of each sample was used to determine VEGF levels. Immunoreactive VEGF was quantified using a sandwich ELISA (Quantikine M Mouse VEGF Immunoassay kit; R and D System, Minneapolis, USA) according to the manufacturer's recommended protocol.

2.9. To Quantify Neutrophil Infiltration. On day 3, the piece of skin from the wound area of animals in each group was 

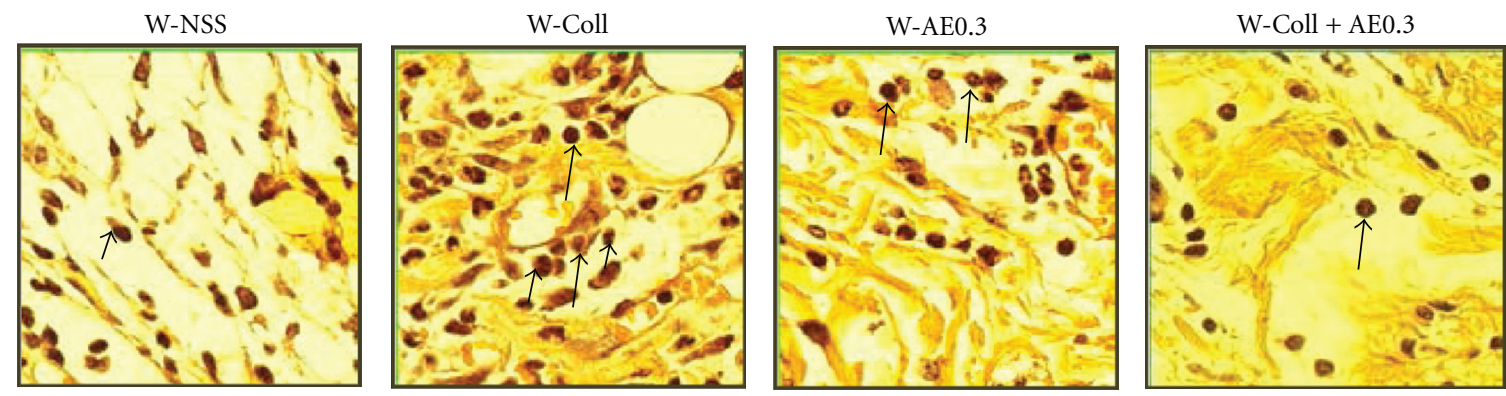

(a)

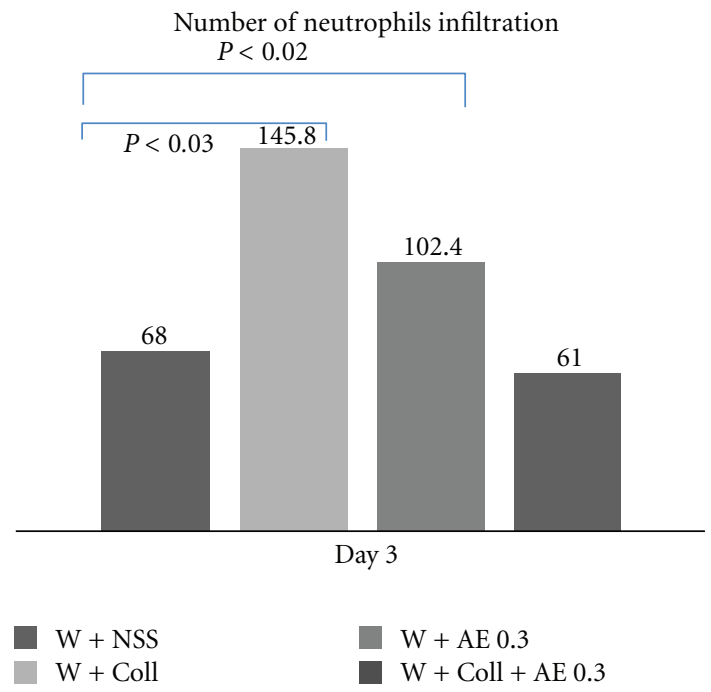

(b)

FIGURE 3: Histochemical analysis of neutrophil filtration within the wounded area on day 3. (a) Arrow indicates the expression of neutrophil infiltration (dark brown color). (b) The means \pm SEM of numbers of infiltrating neutrophils per $0.25 \mathrm{~mm}^{2}$ on day 3 in the W-NSS, W-AE, $\mathrm{W}$-Coll, and W-Coll-AE groups.

fixed in $4 \%$ phosphate-buffered formalin for 2-3 days and then embedded in paraffin. From the paraffin embedded tissue-blocks, $5 \mu \mathrm{m}$ sections were serially cut and stained with hematoxylin and eosin ( $\mathrm{H}$ and $\mathrm{E})$ for routine histology. All histopathology examinations were performed by the same pathologist. The specimens were evaluated for the inflammatory reaction. Under 40x magnification, five areas/slide and three slides/mouse were examined using the Eclipse E600 microscope and the digital camera, DXM1200F [27]. The number of neutrophils within each photomicrograph was then analyzed by image software (Global Lab II).

2.10. Statistical Analysis. Results were expressed as means and standard errors of mean (SEM). For comparison among all groups, one-way analysis of variance (one-way ANOVA) with a post hoc test was used. Significant difference was considered at the probability level ( $P$ value) lower than 0.05 .

\section{Results}

3.1. Extent of Neutrophil Infiltration. On day 3, the results showed that the number of neutrophils infiltrating in the W-NSS group was significantly higher than the sham group
$(P<0.01)$. Neutrophil infiltration was not significantly different after treatment with AE (W-AE0.3) when compared to the W-NSS group. The W-Coll group had the highest numbers of infiltrating neutrophils that were significantly higher than the W-NSS group $(P<0.03)$. In the $\mathrm{W}$ Coll-AE0.3 group, there was no significant difference when compared to the W-NSS group (Figure 3).

3.2. Determination of Wound Closure. On day 7 after the operation, the percent of wound closure in the W-AE0.3 group did not show a significant difference when compared with the W-NSS group. The percent of wound closure in the $\mathrm{W}$-Coll and W-Coll-AE0.3 groups were significantly higher than in the W-NSS group $(P<0.01, P<0.03$, resp. $)$ (Figure 4).

On the day 14, the wound closure of the W-AE0.3, WColl, and W-Coll-AE0.3 groups were significantly increased as compared with the W-NSS group $(P<0.009, P<0.006$, and $P<0.002$, resp.) (Figure 4).

3.3. Determination of Capillary Vascularity. The results on day 7 showed that the $\% \mathrm{CV}$ of the $\mathrm{W}-\mathrm{AE} 0.3$ was significantly increased when compared to the W-NSS group $(P<0.045)$. 
In the combination group, $\mathrm{W}-\mathrm{Coll}-\mathrm{AE} 0.3$, the $\% \mathrm{CV}$ was higher than in the W-NSS group $(P<0.03)$, as shown in Figure 5.

On day 14 , the $\%$ CVs of all treatment groups, W-Coll, W$\mathrm{AE} 0.3$, and W-Coll-AE0.3, were significantly increased when compared with the W-NSS group $(P<0.045)$, as shown in Figure 5.

3.4. Extent of Reepithelialization. The reepithelialization results on day 7 showed no significant differences among all groups (Figure 6). On day 14, the percentage of reepithelialization in the $\mathrm{W}$-Coll and $\mathrm{W}$-Coll-AE0.3 groups were significantly increased when compared to the W-NSS group $(P<0.045)$, as shown in Figure 6.

3.5. Determination of VEGF Levels. The results on day 7 showed that the levels of VEGF in the W-Coll-AE0.3 group were significantly increased when compared to the W-NSS group $(P<0.045)$. However, on day 14 , the results showed that there was no significant difference among the groups except in the levels of VEGF between the W-Coll and W-NSS groups $(P<0.045)$, as shown in Figure 7.

\section{Discussion}

The experiment in this study was conducted to evaluate the effects of the Acanthus ebracteatus Vahl. extract with collagen scaffold on angiogenesis and wound closure in a full-thickness-wound mouse model. The results showed that collagen, in combination with Acanthus ebracteatus Vahl., could increase wound closure in full thickness wounds better than collagen alone or Acanthus ebracteatus Vahl. treatment alone.

As shown in Figure 3, the number of infiltrating neutrophils on day 3 was significantly elevated in wounded mice when compared to the sham group.

As expected, the number of infiltrating neutrophils in the W-Coll group increased because the collagen scaffold is recognized as foreign matter to the body. Interestingly, in the W-Coll-AE0.3 group, the number of infiltrating neutrophils was significantly reduced when compared to the $\mathrm{W}$-Coll group; the number of infiltrating neutrophils was not significantly different from the W-NSS group (Figure 3).

These results indicated that at high doses of $\mathrm{AE}$, the extract can be used to inhibit neutrophil infiltration, which was induced by the implantation of the collagen scaffold within 3 days. It has been previously reported that the bioactive compounds in $\mathrm{AE}$ act as anti-inflammatory agents [1012]. Anti-inflammatory bioactive compounds in $\mathrm{AE}$, including flavonoids, verbascoside, and $\beta$-sitosterol, have been reported. [10-12]. Moreover, the report by Laupattarakasem et al. [4] has shown that Acanthus ebracteatus Vahl. could exert anti-inflammatory effects by reducing thombaxane $\mathrm{B}_{2}$ $\left(\mathrm{TXB}_{2}\right)$ and leukotriene $\mathrm{B}_{4}\left(\mathrm{LTB}_{4}\right)$. Therefore, it is suggested that the topical $\mathrm{AE}$ application with the collagen scaffold might be beneficial in clinical use and, in particular, may help reduce the oral antibiotic requirements.

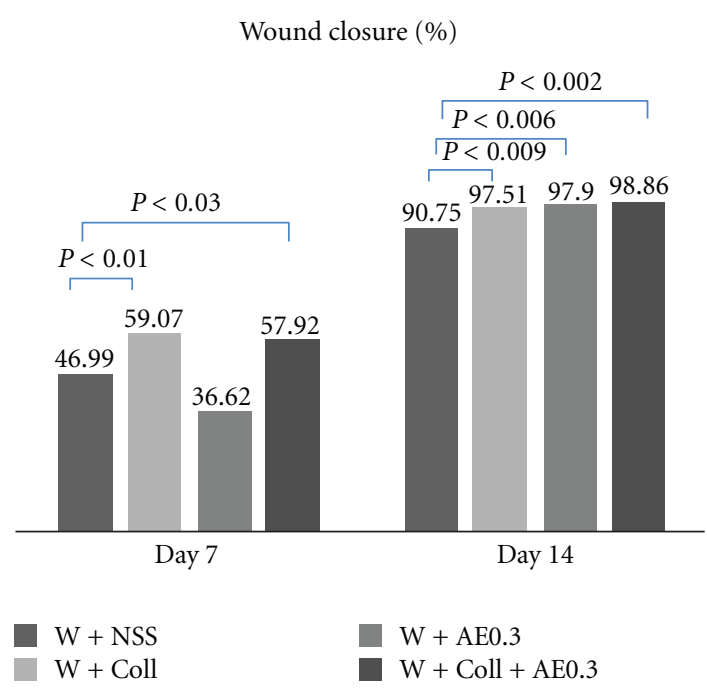

Figure 4: Percentages of wound closure in the W-NSS, W-AE, WColl, and W-Coll-AE groups were determined on days 7 and 14 after operation (mean $\pm \mathrm{SEM}) .{ }^{*} P<0.05$ compared with control group. NS (nonsignificantly) compared with control.

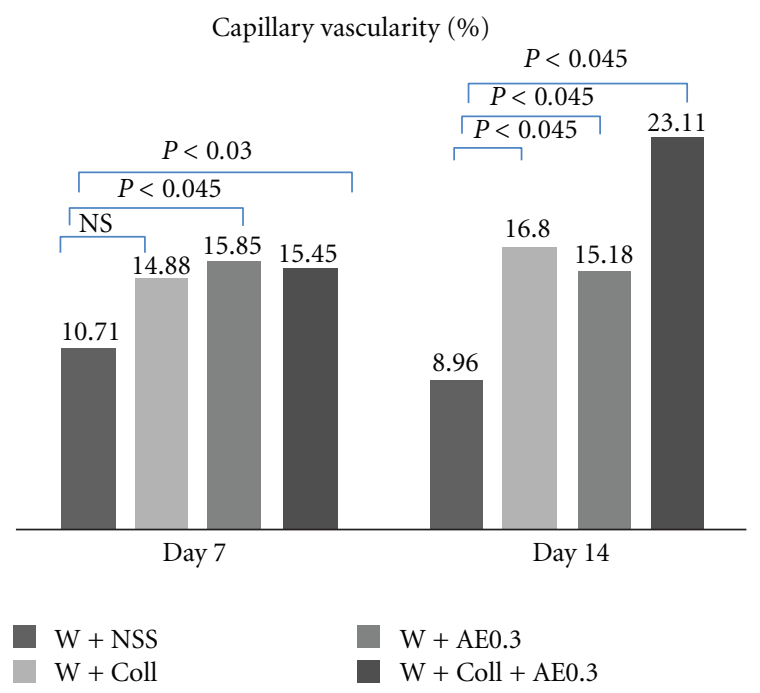

Figure 5: Capillary vascularity (CV) measured at the different timepoints (days 7 and 14) in the W-NSS, W-AE, W-Coll, and WColl-AE groups. Values are expressed as mean \pm SEM.

The present study also suggested that increasing angiogenesis was an essential process in enhancing wound closure. Figure 7 showed that on day 7, the \%CVs of the $\mathrm{W}$ Coll, W-AE0.3, and W-Coll-AE0.3 groups were significantly increased when compared to the W-NSS group. However, the $\% \mathrm{CV}$ of the W-Coll-AE0.3 group showed the most significant increase on day 14 . Therefore, AE0.3 may enhance bovine collagen scaffold action on wound healing in association with its potential to mediate angiogenesis.

There are several reports that indicated that a bioactive compound in $\mathrm{AE}, \beta$-sitosterol, could act as an effective angiogenic factor $[10,28]$. $\beta$-sitosterol can be dissolved in ethanol; therefore, our AE ethanol extract should definitely 
W-NSS

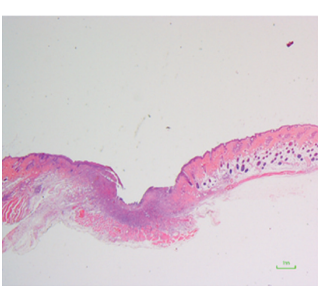

W-AE0.3

Day 7
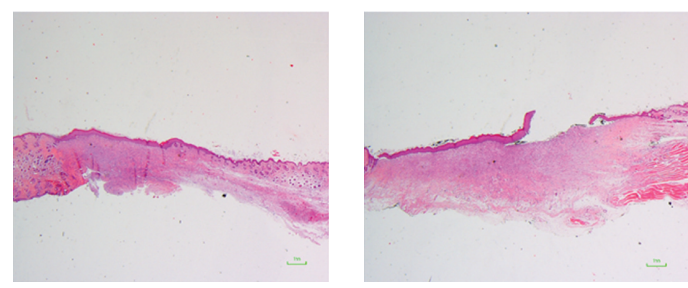

(a)

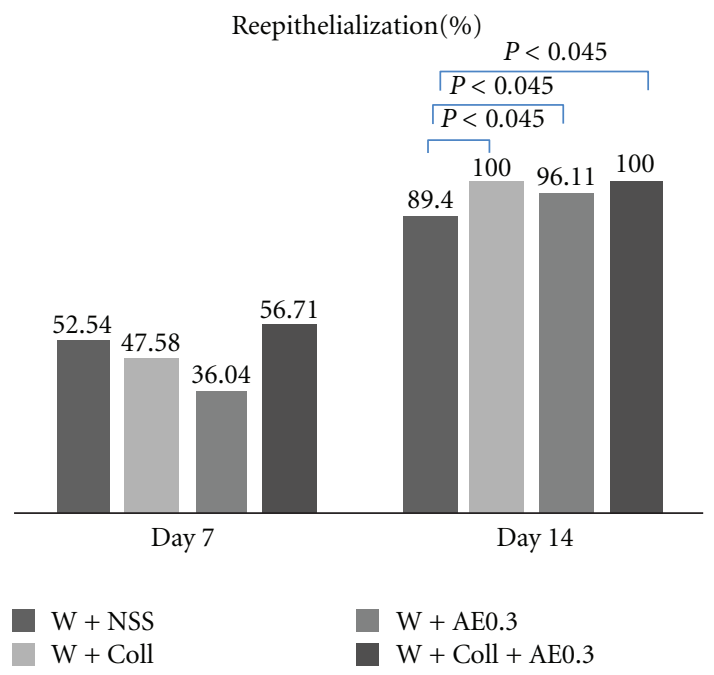

(b)
$\mathrm{W}+$ Coll $+\mathrm{AE} 0.3$
$\mathrm{W}-\mathrm{Coll}+\mathrm{AE} 0.3$

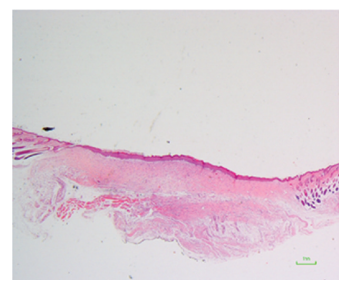

-

Figure 6: (a) Reepithelialization on day 7. (b) Means \pm SEM of wound area of the W-NSS, W-AE, W-Coll, and W-Coll-AE groups (d) on days 7 and 14.

contain some $\beta$-sitosterol, although the level of it in the extract used in this study was not determined.

The present results also show that on day 7, tissue VEGF levels were significantly increased in the $\mathrm{W}$-Coll-AE0.3 group when compared to the W-NSS group (Figure 7). According to Choi et al. [28], $\beta$-sitosterol in the AE extract could enhance angiogenesis by increasing VEGF and VEGFR (Flk1) in vitro. Therefore, we believe that the effect of $\mathrm{AE}$ on increasing wound angiogenesis is associated with its active ingredient, $\beta$-sitosterol, that upregulates VEGF expression.

Several studies have reported that angiogenesis is an essential process for wound healing, and the expression of VEGF is essential for angiogenesis [29-31]. Our results demonstrate that VEGF is increased significantly during the 7 days prior to wound neovascularization. However, VEGF expression started to decline on day 14 when the $\% \mathrm{CV}$ is increased, particularly, in the $\mathrm{W}$-Coll-AE0.3 group.

We have performed a further study to define the correlation between wound closure and capillary vascularity. By using mean values from each group, as shown in Figure 8, the correlation between capillary vascularity ( $x$-axis) was significantly correlated with wound closure $(y)$ and best fitted with a linear line; $y=0.5841 x+86.8870\left(R^{2}=0.79\right)$. This linear correlation confirmed that wound closure was increased in relation to the increase in wound capillary density.

In the normal process of wound healing, epithelialization represents the final stage of the proliferative phase, which occurs between day 3 and day 14. In our study, the process of reepithelialization was assessed on day 7 and day 14. The reepithelialization was not significantly different between all groups on day 7 but was significantly increased on day 14, particularly for the W-Coll and W-Coll-AE0.3 groups when compared to the WNSS group (Figure 6). In fact, collagen is an important factor in the formation of the extracellular matrix, which is an ultrastructure that supplies a substratum for cell adhesion. It also plays a critical role in helping to regulate the growth, movement, and differentiation of cells throughout its network [32]. The present results provide evidence about the roles of the collagen scaffold in cell migration. The results on day 14 indicated that reepithelialization was effectively enhanced up to $100 \%$ in the W-Coll and W-Coll-AE0.3 groups. The mechanism underlying these observations may be associated with the effects on angiogenesis, which is the other important process that is required for wound healing, as discussed above. 


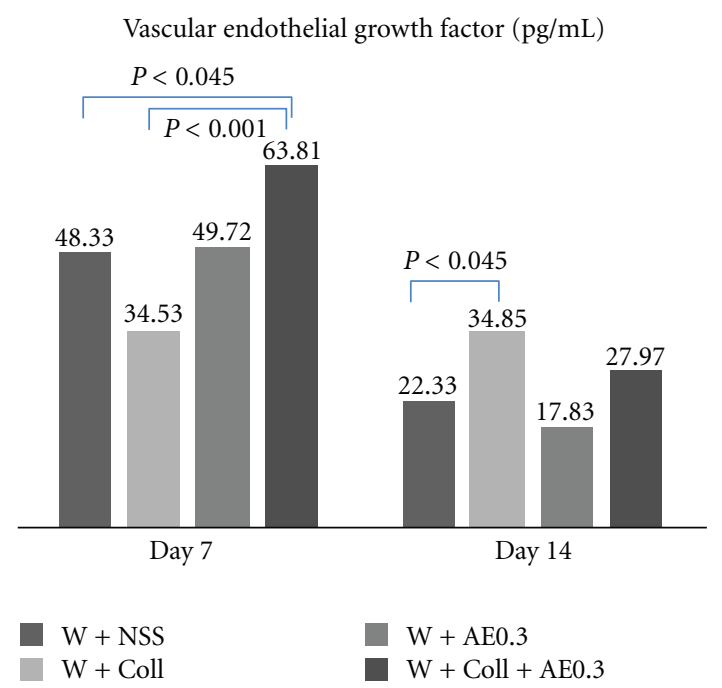

FIGURE 7: Tissue VEGF $(\mathrm{pg} / \mathrm{mL})$ of each group was determined on days 7 and 14 in the W-NSS, W-Coll, W-AE, and W-Coll-AE groups.

Relationship between capillary vascularity $(\mathrm{CV})$ and wound closure

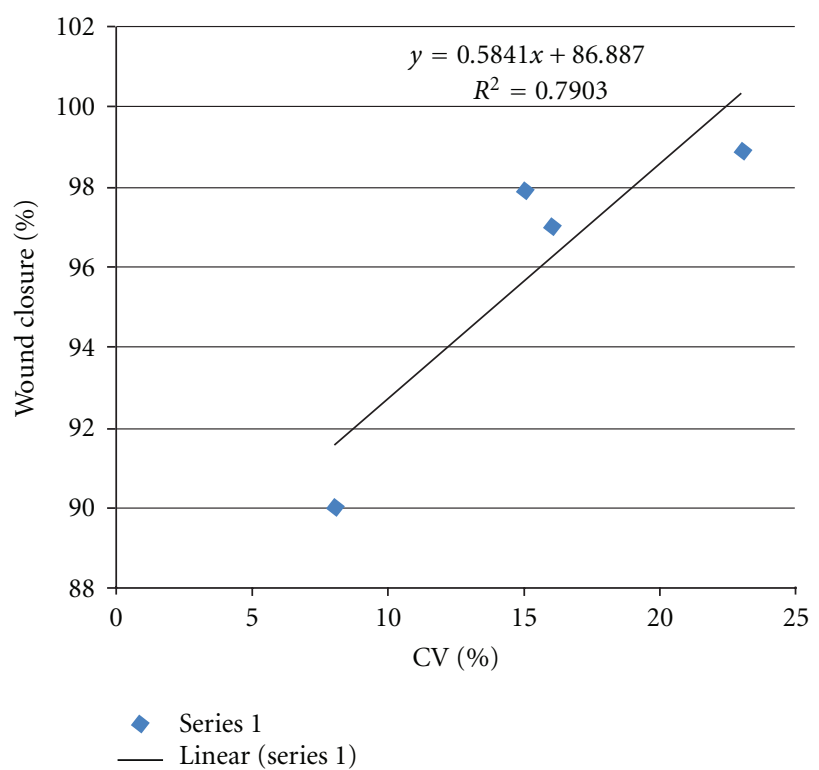

Figure 8: Relationship between capillary vascularity $(\mathrm{CV})$ and wound closure. Data were taken from mean of each group on day 14.

In conclusion, daily topical treatment with $\mathrm{AE}(0.3 \mathrm{~g} / \mathrm{kg}$ BW) could enhance the beneficial effects of the collagen scaffold that is implanted in full-thickness wounds. Possible mechanisms underlying wound healing might involve the combined effects of collagen and $\mathrm{AE}$ to decrease wound inflammation, increase VEGF expression, stimulate the angiogenesis, and enhance reepithelialization.

\section{Acknowledgments}

This study was supported by Ratchadapiseksompotch Fund of the Faculty of Medicine, the 90th Anniversary Research Fund, and the Graduate School, Chulalongkorn University, Thailand. Our gratitude also goes to Dr. H. Niimi for his useful advice.

\section{References}

[1] S. M. Bauer, R. J. Bauer, and O. C. Velazquez, "Angiogenesis, vasculogenesis, and induction of healing in chronic wounds," Vascular and Endovascular Surgery, vol. 39, no. 4, pp. 293-306, 2005.

[2] M. G. Tonnesen, X. Feng, and R. A. F. Clark, "Angiogenesis in wound healing," Journal of Investigative Dermatology Symposium Proceedings, vol. 5, no. 1, pp. 40-46, 2000.

[3] W. William and W. Vincent, "Angiogenesis in wound healing," in Supplement to Contemporary Surgery, pp. 1-36, Dowden Health Media, 2003.

[4] P. Laupattarakasem, P. J. Houghton, J. R. S. Hoult, and A. Itharat, "An evaluation of the activity related to inflammation of four plants used in Thailand to treat arthritis," Journal of Ethnopharmacology, vol. 85, no. 2-3, pp. 207-215, 2003.

[5] A. E. Sabine, K. Thomas, and M. D. Jeffrey, "Inflammation in wound repair: molecular and cellular mechanisms," Journal of Investigative Dermatology, vol. 127, pp. 514-525, 2007.

[6] H. N. Subudhi, B. P. Choudhury, and B. C. Acharya, "Some potential medicinal plants of Mahanadi delta in the state of Orissa," Journal of Economic and Taxonomic Botany, vol. 16, no. 2, pp. 479-487, 1992.

[7] L. M. Perry, Medicinal Plants of East and Southeast Asia Attributed Properties and Use, vol. 2, The Massachusetts Institute of Technology Press, London, UK, 1980.

[8] I. Burkill, Dictionary of the Economic Products of the Malay PeninsulaMalaysia, Kuala Lumpur, vol. 27, Ministry of Agriculture and Cooperatives, Kuala Lumpur, Malaysia, 1966.

[9] S. Pongboonrod, Medicinal Plants of Thailand, Kasem Bunnakit Publishing, Bangkok, Thailand, 1971.

[10] M. Rücker, M. W. Laschke, D. Junker et al., "Angiogenic and inflammatory response to biodegradable scaffolds in dorsal skinfold chambers of mice," Biomaterials, vol. 27, no. 29, pp. 5027-5038, 2006.

[11] B. N. Shah, A. K. Seth, and K. M. Maheshwari, "A review on medicinal plants as a source of anti-inflammatory agents," Research Journal of Medicinal Plant, vol. 5, no. 2, pp. 101-115, 2011.

[12] P. Siripong, K. Boonsong, and K. Kwanjai, "Study on antitumor potential of A. ebracteatus Vahl. Roots," Thai Cancer Journal, vol. 24, pp. 29-39, 1998.

[13] W. Kongcharoensuntorn, N. Chomosot, J. Jindamol et al., "Screening of some Thai medicinal plants for antimicrobial activity and antioxidant activity against microorganisms," in Proceedings of the 31st Congress on Science and Technology of Thailand, Suranaree University of Technology, October 2005.

[14] A. Ghosh, S. Misra, A. K. Dutta, and A. Choudhury, "Pentacyclic triterpenoids and sterols from seven species of mangrove," Phytochemistry, vol. 24, no. 8, pp. 1725-1727, 1985.

[15] S. Misra, A. K. Datta, S. Chattopadhyay, and A. Ghosh, "Hydrocarbons and wax esters from seven species of mangrove leaves," Phytochemistry, vol. 26, no. 12, pp. 3265-3268, 1987. 
[16] E. J. Moon, Y. M. Lee, O. H. Lee et al., "A novel angiogenic factor derived from Aloe vera gel: $\beta$-sitosterol, a plant sterol," Angiogenesis, vol. 3, no. 2, pp. 117-123, 1999.

[17] S. Hokputsa, S. E. Harding, K. Inngjerdingen et al., "Bioactive polysaccharides from the stems of the Thai medicinal plant Acanthus ebracteatus: their chemical and physical features," Carbohydrate Research, vol. 339, no. 4, pp. 753-762, 2004.

[18] J. Z. Wang, H. Tsumura, K. Shimura, and H. Ito, "Antitumor activity of polysaccharide from a Chinese medicinal herb, Acanthopanax giraldii Harms," Cancer Letters, vol. 65, no. 1, pp. 79-84, 1992.

[19] C. C. Shih, M. K. Lu, J. J. Cheng, and L. W. Danny, "Antiangiogenic activities of polysaccharides isolated from medicinal fungi," FEMS Microbiology Letters, vol. 249, no. 2, pp. 247-254, 2005.

[20] M. Sandoval, N. N. Okuhama, X. J. Zhang et al., "Antiinflammatory and antioxidant activities of cat's claw (Uncaria tomentosa and Uncaria guianensis) are independent of their alkaloid content," Phytomedicine, vol. 9, no. 4, pp. 325-337, 2002.

[21] M. Kremer, E. Lang, and A. Berger, "Evaluation of dermalepidermal skin equivalents ("composite-skin") of human keratinocytes in a collagen-glycosaminoglycan matrix (Integra(TM) artificial skin)," British Journal of Plastic Surgery, vol. 53, no. 6, pp. 459-465, 2000.

[22] J. Somchaichana, Effect of Acanthus ebractetus Vahl. Extract in combination with collagen scaffold on angiogenesis and wound closure in mice skin model [M.S. thesis], Inter- Department of Physiology, Chulalongkorn University, Bangkok, Thailand, 2000.

[23] S. Piyaviriyakul, P. Kupradinun, and S. Tunsakul, "Chronic toxicity of Acanthus ebracteatus Vahl. in rat," in Proceedings of the 6th National Cancer Conference, Bangkok, Thailand, 2001.

[24] B. M. Hall, "Cells mediating allograft rejection," Transplantation, vol. 51, no. 6, pp. 1141-1151, 1991.

[25] J. K. HeimbachLim, L. Saliba, and M. J. Smith, "Normal saline wound dressing-is it really normal?" British Journal of Plastic Surgery, vol. 53, pp. 42-45, 2000.

[26] S. Viboolvorakul, H. Niimi, N. Wongeak-in, S. Eksakulkla, and S. Patumraj, "Increased capillary vascularity in the femur of aged rats by exercise training," Microvascular Research, vol. 78, no. 3, pp. 459-463, 2009.

[27] A. E. Sakallioğlu, Ö. Başaran, B. H. Özdemir, Z. Arat, M. Yücel, and M. Haberal, "Local and systemic interactions related to serum transforming growth factor- $\beta$ levels in burn wounds of various depths," Burns, vol. 32, no. 8, pp. 980-985, 2006.

[28] S. Choi, K. W. Kim, J. S. Choi et al., "Angiogenic activity of $\beta$-sitosterol in the ischaemia/reperfusion-damaged brain of Mongolian gerbil," Planta Medica, vol. 68, no. 4, pp. 330-335, 2002.

[29] D. R. Knighton, G. D. Phillips, and V. D. Fiegel, "Wound healing angiogenesis: indirect stimulation by basic fibroblast growth factor," Journal of Trauma, vol. 30, no. 12, supplement, pp. S134-S144, 1990.

[30] S. S. Brem, P. M. Gullino, and D. Medina, "Angiogenesis: a marker for neoplastic transformation of mammary papillary hyperplasia," Science, vol. 195, no. 4281, pp. 880-882, 1977.

[31] L. E. Huang, Z. Arany, D. M. Livingston, and H. F. Bunn, "Activation of hypoxia-inducible transcription factor depends primarily upon redox-sensitive stabilization of its $\alpha$ subunit," The Journal of Biological Chemistry, vol. 271, no. 50, pp. 32253-32259, 1996.

[32] E. Stuart and J. David, "Basic science of wound healing," Surgery, vol. 23, pp. 37-42, 2005. 

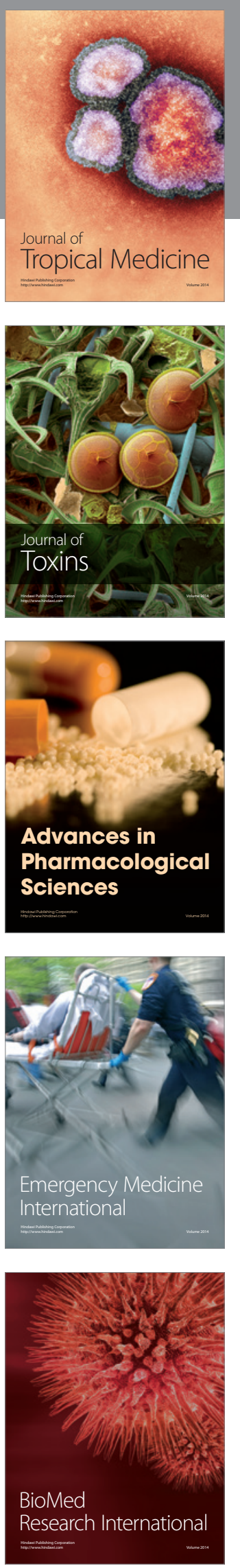
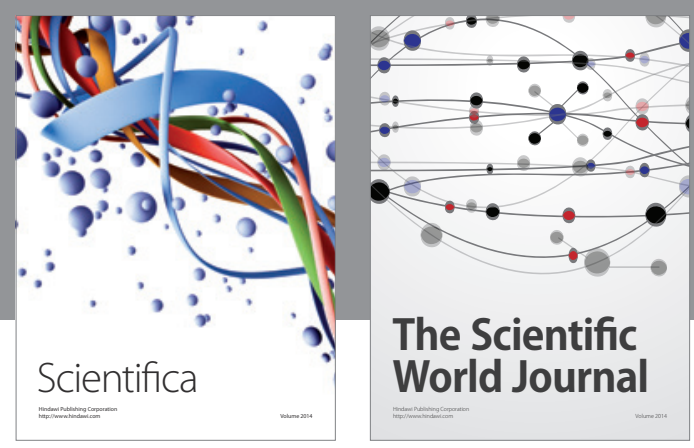

The Scientific World Journal
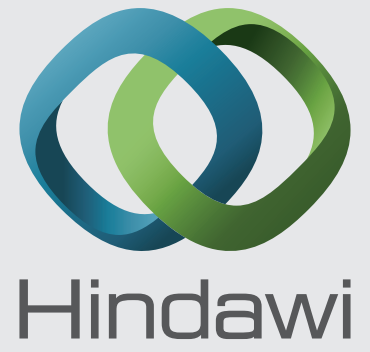

Submit your manuscripts at

http://www.hindawi.com
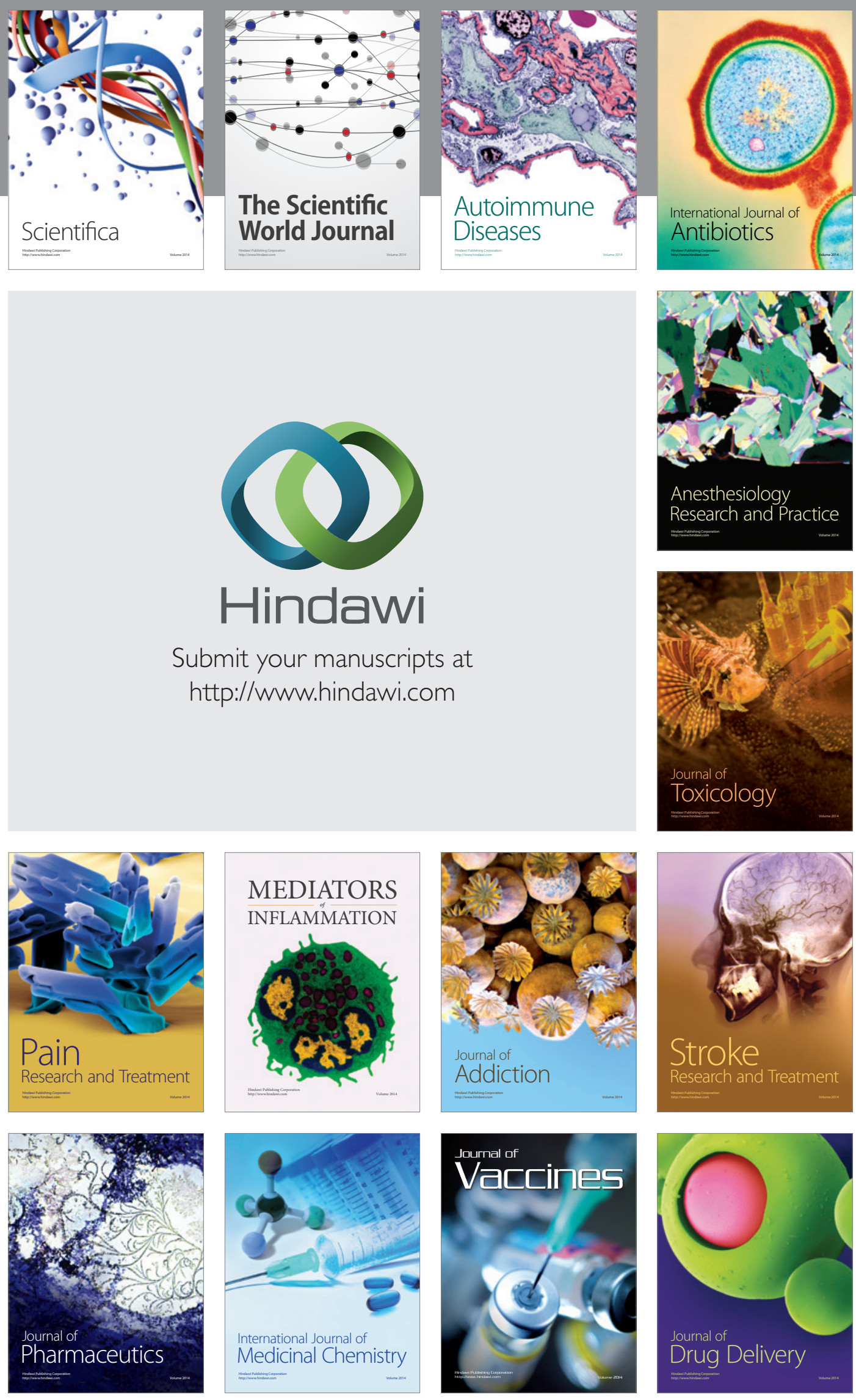